
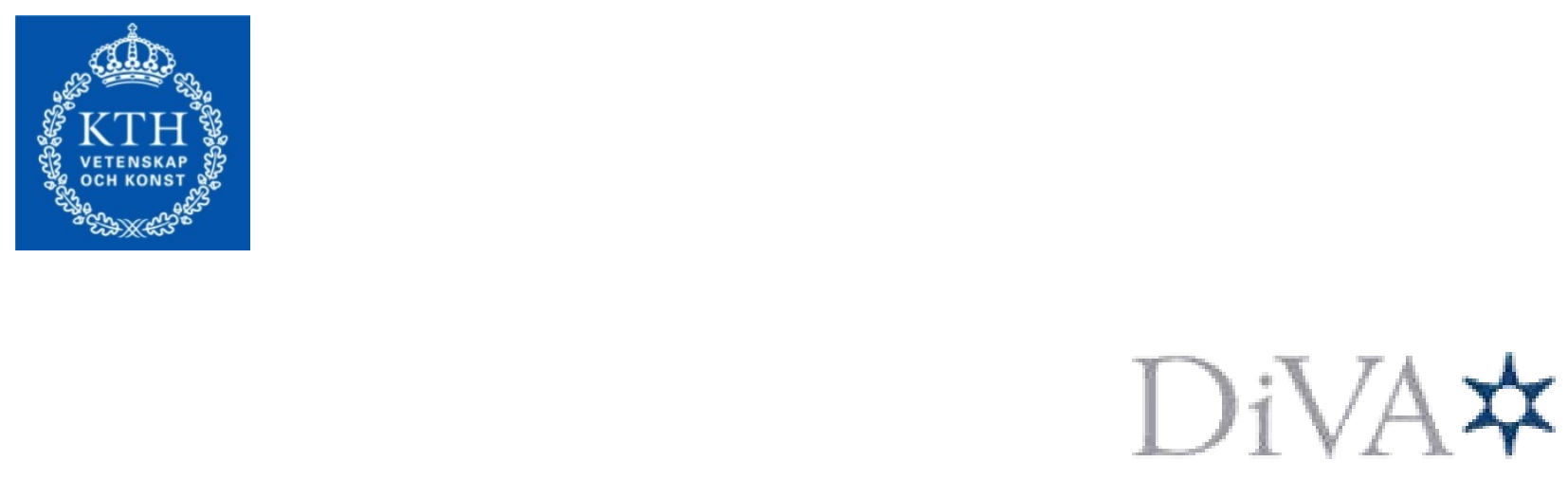

http://kth.diva-portal.org

This is an author produced version of a paper presented at The 11th IEEE International NEWCAS Conference,June 16 - 19, 2013, Paris. This paper has been peer-reviewed but does not include the final publisher proof-corrections or proceedings pagination.

(C) 2013 IEEE. Personal use of this material is permitted. Permission from IEEE must be obtained for all other uses, in any current or future media, including reprinting/republishing this material for advertising or promotional purposes, creating new collective works, for resale or redistribution to servers or lists, or reuse of any copyrighted component of this work in other works.

Citation for the published paper:

Sha Tao, Saul Rodriguez and Ana Rusu.

DAC Waveform Effects in CT Incremental $\Sigma \Delta$ ADCs for Biosensor Applications

Proceedings of The 11th IEEE International NEWCAS Conference, June 16 - 19, 2013, Paris.

Access to the published version may require subscription.

Published with permission from: IEEE 


\title{
DAC Waveform Effects in CT Incremental $\Sigma \Delta$ ADCs for Biosensor Applications
}

\author{
Sha Tao, Saul Rodriguez and Ana Rusu \\ School of Information and Communication Technology (ICT) \\ KTH Royal Institute of Technology \\ SE-164 40 Kista, Sweden \\ Email:\{stao,saul,arusu\}@kth.se
}

\begin{abstract}
Incremental sigma-delta (I $\Sigma \Delta)$ analog-to-digital converters (ADCs), which are essentially $\Sigma \Delta$ ADCs with periodic resetting, are well suited for low-power low-speed biosensor applications. In recent years, the potential advantage in terms of power dissipation of continuous-time (CT) I $\Sigma \Delta$ ADCs have been explored. This paper analyzes the impact of feedback digitalto-analog converters (DACs) on the performance of CT I $\Sigma \Delta$ ADCs. Different feedback DAC schemes are firstly analyzed and then evaluated by employing them in a $3^{\text {rd }}$ order singlebit CT I $\Sigma \Delta$ ADC. Simulation results are discussed considering the trade-off between the timing error sensitivity and the power consumption, thereby offering a reference for selecting a power efficient feedback DAC scheme.
\end{abstract}

\section{INTRODUCTION}

Unlike conventional $\Sigma \Delta$ ADCs that process input signals continuously, incremental $\Sigma \Delta$ ADCs reset their memory elements before each conversion, and hence can offer sampleby-sample conversions as Nyquist-rate ADCs [1]. Historically, incremental $\Sigma \Delta$ ADCs have been developed for instrumentation and measurement (I\&M) applications. Among these applications, biosensors, which typically feature a low frequency input signal from a few hertz to a few kilo hertz [2], is a recent research hot spot. Compared with traditional I\&M applications, biosensors are more power-constrained since they are mostly powered by small battery or even self-powered by harvested energy.

Majority of the published works on incremental $\Sigma \Delta(\mathrm{I} \Sigma \Delta)$ ADCs employ discrete-time (DT) loop filters. Only recently, continuous-time (CT) implementations have been explored, with the aim of reducing the power consumption. Similar to traditional CT $\Sigma \Delta$ ADCs, CT I $\Sigma \Delta$ ADCs have relaxed settling and bandwidth requirements on their integrators [3]. As in the CT $\Sigma \Delta$ ADCs, the CT $\Sigma \Delta$ ADCs suffer from similar design challenges, e.g., the increased sensitivity to nonidealities in the feedback DACs compared with their DT counterparts. The most critical DAC nonidealities are timing errors that alter the width and position of the feedback DAC waveforms, known as clock jitter and excess-loop-delay (ELD) [4].

In literature, there are theoretical and experimental analysis on the DAC timing error sensitivity of CT $\Sigma \Delta$ modulators [59]. However, for low-power low-speed applications, CT I $\Sigma \Delta$ ADCs have specific challenges which need to be addressed and analyzed. Firstly, even for the relatively low sampling frequency used in these applications, the tolerance range of timing error in feedback DACs needs to be identified to ensure a robust design. Moreover, the resetting operation in $I \Sigma \Delta$ ADCs would introduce some issues and differences compared with the $\Sigma \Delta$ ADCs. Most importantly, the power consumption is a big concern in biosensors and thus some design trade-offs should be considered.

In this paper, the sensitivity to timing errors of representative feedback DAC schemes in CT I $\Sigma \Delta$ ADCs are analyzed. Additionally, their impact on speed and bandwidth requirements for the active blocks in the modulator are examined. The paper is organized as follow. Section II introduces the feedback DAC schemes included in this study. The sensitivity to jitter and ELD is analyzed, and the differences induced by the incremental mode of operation are illustrated. The impact of various DAC schemes on the $1^{\text {st }}$ integrator are also presented. In section III, a CT I $\Sigma \Delta$ ADC, which employs different DAC schemes, is simulated using Matlab/Simulink and Verilog-A models. These results are compared and discussed considering low-power low-speed biosensor applications. Section IV finally concludes the paper.

\section{DAC Nonidealities IN CT I $\Sigma \Delta$ ADCs}

The $3^{\text {rd }}$ order single-bit CT I $\Sigma \Delta$ ADC [3] given in Fig. 1 is used as a demonstration vehicle for this analysis. The input signal is sampled at the rate of $f_{S} / M$ and then held for the entire conversion cycle, $T_{S} \times M$. The modulator runs at the clock rate of $f_{S}$ and its integrators are reset every $M$ clock cycles. At the end of each conversion, the modulator output is digitized by the digital filter which is also reset every $M$ clock cycles. Here, the number of clock cycles in each conversion, $M$, is equivalent to the oversampling ratio (OSR) in conventional $\Sigma \Delta$ ADCs.

The sensitivity to clock jitter is one of the most significant DAC nonidealities from which the CT implementation suffers. The jitter sensitivity depends on the waveform of feedback DAC scheme, as shown in Fig. 1. The selected feedback DACs can be divided into two categories: the commonly used rectangular DACs including non-return-to-zero (NRZ), halfreturn-to-zero (HRZ), and return-to-zero (RZ) [5]; and the exponentially decaying DACs including switched-capacitorresistor (SCR), full clock period SCR (FSCR), and switchedcapacitor switched-resistor (SCSR) [6-8]. For a single loop CT $\Sigma \Delta$ modulator with a single-bit DAC, the jitter induced 


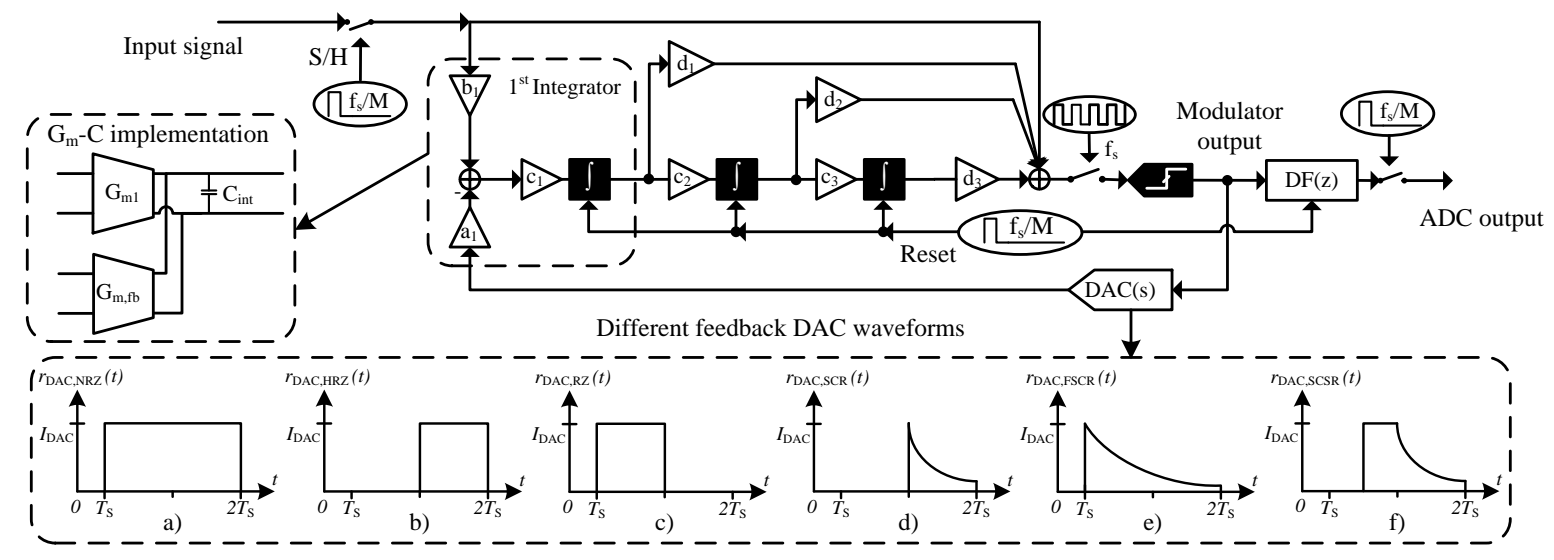

Fig. 1. Block diagram of a $3^{\text {rd }}$ order 1-bit CT I $\Sigma \Delta$ ADC with different feedback DAC schemes. a) NRZ, b) HRZ, c) RZ, d) SCR, e) FSCR, f) SCSR.

in-band-noise (IBN) can be estimated as [9]:

$$
\mathrm{IBN}_{\sigma_{j}} \approx\left(\frac{a_{1}}{b_{1}}\right)^{2} \frac{A}{\mathrm{OSR}} \frac{\left(P \cdot I_{D A C}\right)^{2}\left(\mathrm{e}^{-\frac{(\beta-\gamma) T_{S}}{\tau}}\right)^{2} \sigma_{j}^{2}}{T_{S}{ }^{2}},
$$

where $a_{1}$ is the feedback coefficient of the corresponding DAC and $b_{1}$ is the feedforward coefficient from the modulator input. All DAC waveforms are assumed to transfer the same amount of feedback charge per clock period, and thus we have $a_{1} / b_{1}=1$ for NRZ DAC, $a_{1} / b_{1}=2$ for HRZ and RZ DACs, and

$$
\frac{a_{1}}{b_{1}}=\frac{1}{-\alpha-\tau \mathrm{e}^{-\frac{(\beta-\gamma)}{\tau}}+\gamma+\tau}
$$

for exponentially decaying DACs. $A$ is the activity factor that is around 0.7 for NRZ and equals 2 for others [5]. $I_{D A C}$ is the normalized peak amplitude of each DAC pulse, and $P$ denotes the pulse transition amplitude factor which equals 2 for NRZ and 1 for the others. $\alpha$ and $\beta$ are the normalized starting and ending time instants of the DAC pulse, and $\gamma$ is the normalized time instant when the exponential decaying begins. $\tau$ is the time constant of the exponential decaying. $\sigma_{j}$ is the clock jitter standard deviation. For rectangular DACs, $\gamma=\beta$ and $\tau \rightarrow \infty$. For exponentially decaying DACs, $\tau=0.2 T_{S}$. For SCR and SCSR, $\gamma=0.5 T_{S}$, while for FSCR $\gamma=0$. In this study, only $\alpha$ and $\beta$ are jitter affected.

Another timing error that affects the performance of the CT implementation is the ELD. The constant delay $t_{d}$ can be normalized as $\tau_{d}=t_{d} / T_{S}$. For HRZ, RZ, SCR, and SCSR DACs, only coefficient mismatch is induced, which mainly leads to increased IBN [4], as long as their delayed waveforms don't exceed the next sampling instant, i.e., $\beta+\tau_{d}<\alpha+$ $T_{S}$. For NRZ and FSCR DACs, on the other hand, any delay in their waveforms would alter the impulse response of the DAC, and consequently the transfer function of the loop filter. When $\tau_{d}>0$, the order of the equivalent DT modulator is incremented, which leads to reduced stability and degraded noise shaping performance $[4,5]$. The ELD mainly stems from the non-zero time for the quantizer to generate outputs and the finite response time of the DAC. The value of $\tau_{d}$ depends on the speed of the transistor, $f_{T}$, the clock sampling frequency,
$f_{S}$, and the number of transistors in the feedback path, $n_{t} . \tau_{d}$ can be estimated as [5]:

$$
\tau_{d} \approx \frac{n_{t} \times f_{S}}{f_{T}} .
$$

Considering the typical frequency bandwidth of biosensor signals (i.e., less than few $\mathrm{kHz}$ ), $f_{S}$ is on the order of hundreds of $\mathrm{kHz}$. Also considering a power efficient design, the transistors are generally biased at the operation point where $f_{T}$ is in the range of tens of $\mathrm{MHz}$ to a few $\mathrm{GHz}$. By rough estimation, $f_{S} / f_{T}$ would be very small, i.e., less than $1 \%$. Typically, only a few transistors would switch in the feedback path. Thus, $n_{t} \ll 10$ and $\tau_{d}$ should be less than $10 \%$.

For I $\Sigma \Delta$ modulator, a resetting cycle appears periodically every $M$ clock cycles, as depicted in Fig.2 (a). For DACs that starts immediately at the rising edge of the clock, i.e., the NRZ, RZ and FSCR DACs, the resetting operation may induce error under certain circumstances. For instance, as shown in Fig. 2 (b), when the NRZ DAC waveform is only affected by clock jitter, there is a $50 \%$ chance that the next rising edge of the jittered clock takes a sample before the next modulator output data arrives, i.e., a zero-value is sampled from the resetting period, $2 T_{S} \sim 3 T_{S}$, instead of the expected sample from $3 T_{S} \sim 4 T_{S}$. Hence, a large error with an integrated area of the entire clock period, $T_{S} \times V_{\text {ref }}$, is introduced. Taking this error into account, the jitter induced IBN for NRZ DAC operating in the incremental mode can be estimated as:

$$
\begin{aligned}
\mathrm{IBN}_{\sigma_{j}}^{N R Z}[d B] & \approx \frac{M}{M+1} 10 \log _{10}\left[\frac{\left(2 \cdot I_{D A C}\right)^{2}{\sigma_{j}}^{2}}{T_{S}{ }^{2}} \frac{0.7}{\mathrm{M}}\right] \\
& +\frac{1}{M+1} 10 \log _{10}\left[\frac{\left(I_{D A C}\right)^{2} \cdot T_{S}{ }^{2}}{T_{S}{ }^{2}} \frac{0.5}{\mathrm{M}}\right]
\end{aligned}
$$

where the second term denotes the noise contribution from the resetting cycle. On the other hand, the DAC waveforms that use only the second half of the clock period, e.g., the SCR and HRZ DACs, do not suffer from such an error, as long as their jittered edge varies less than $50 \% T_{S}$. Generally, DACs that start at a certain time instance, e.g., the SCSR DAC where $\alpha \gg \sigma_{j}$, are free from this error. Similarly, as long as the ELD 


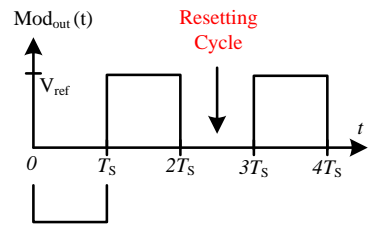

a)

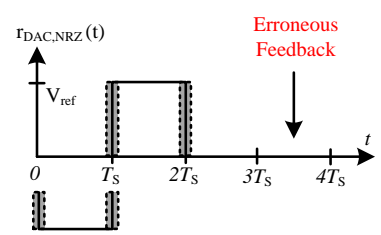

b)
Fig. 2. Impact of the resetting cycle on DAC waveforms with jittered clock. (a) modulator output with resetting, and (b) erroneous feedback of NRZ DAC.

of the DAC is larger than the variation of the clock jitter, the aforementioned error can be avoided for all DAC schemes.

It is also worth mentioning that in most cases the I $\Sigma \Delta \mathrm{ADC}$ achieves lower signal-to-quantization-noise-ratio (SQNR) than traditional $\Sigma \Delta$ ADC. It has been illustrated in [10] that an $\mathrm{I} \Sigma \Delta$ ADC has higher in-band quantization noise than a $\Sigma \Delta$ ADC with the same modulator order and OSR. The increased quantization noise floor in the incremental case, in turn, relaxes the requirement on jitter and ELD induced IBN.

As it can be indicated from Eq. (1), the charge injected by the exponentially decaying DACs is less sensitive to clock jitter. However, we can appreciate from Eq. (2) that their feedback coefficient $a_{1}$ would be significantly larger than for rectangular DACs to transfer the same amount of charge. The increased coefficient gives rise to a linear error if the integrator has finite gain bandwidth (GBW); a non-linear error when the integrating op-amp is slew-rate (SR) limited. The integrator gain error (GE) induced by finite GBW and the minimum required op-amp SR can be respectively expressed as [4,6]:

$$
\begin{gathered}
\mathrm{GE}=\frac{\mathrm{GBW}}{\left(a_{1}+b_{1}\right) \cdot c_{1} \cdot f_{S}+\mathrm{GBW}}, \\
\mathrm{SR}\left[\frac{V}{S}\right] \geq f_{S}\left(b_{1} \cdot c_{1} \cdot u+a_{1} \cdot c_{1} \cdot V_{r e f}\right),
\end{gathered}
$$

where $u$ is the input signal amplitude and $V_{\text {ref }}$ is the DAC reference voltage. It can be appreciated from Eq. (5) and (6) that the larger the feedback coefficient $a_{1}$ is, the higher GBW and SR requirements are imposed on integrators.

\section{Simulation Results And Discussions}

The theoretical analysis is complemented by behavioral simulations of the $3^{\text {rd }}$ order 1-bit CT I $\Sigma \Delta$ ADC (shown in Fig. 1) where different schemes are employed for the feedback DAC. The modulator is reset before processing each sampled input and then runs continuously for $\mathrm{M}$ cycles. Considering a typical biosensor application which features a resolution of 10-bit and an input signal bandwidth of $4 \mathrm{kHz}, M=40$ is chosen in simulations. The CT loop filter coefficients $c_{1}, c_{2}$ and $c_{3}$ are derived for different feedback DACs by using the impulse-invariant transformation (IIT). For each DAC scheme, IIT is also used to obtain the transfer function of the digital filter, $D F(z)$, following the method described in [3].

The DAC's sensitivity to timing error and its impact on the signal-to-noise-and-distortion ratio (SNDR) performance

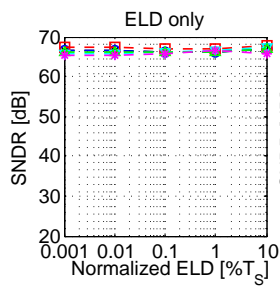

(a)

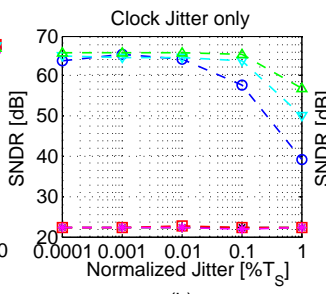

(b)

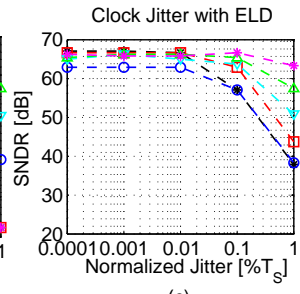

(c)
Fig. 3. Simulated SNDR of the CT I $\Sigma \Delta$ ADC under the influence of timing errors. (a) SNDR vs. $\tau_{d}$, (b) SNDR vs. $\sigma_{j}$, and (c) SNDR vs. $\sigma_{j}$ for $t_{d}=$ $10 \% T_{S} \cdot[\times: \mathrm{RZ}, \circ: \mathrm{HRZ}, \square: \mathrm{NRZ}, \nabla: \mathrm{SCR}, \triangle: \mathrm{SCSR}, *: F S C R$.

are evaluated in Matlab/Simulink environment. The CT I $\Sigma \Delta$ ADC employing different DAC schemes is firstly simulated under the influence of ELD. As explained in sec. II, $\tau_{d}$ can hardly exceed $10 \%$ according to Eq. (3). Fig. 3 (a) shows that no significant variation in SNDR is observed with the $t_{d}$ swept up to $10 \% T_{S}$. Thus, ELD does not play a deterministic role in this application. The CT I $\Sigma \Delta$ ADC is then simulated under the influence of clock jitter. The amount of jitter is varied in the range of $\sigma_{j}=0.0001 \% T_{S} \sim 1 \% T_{S}$, corresponding to $3.125 \mathrm{ps} \sim 31.25 \mathrm{~ns}$. This root-mean-squared jitter range is readily achievable when the sampling clock is derived directly from crystal oscillators. Fig 3 (b) shows the simulated SNDR with only clock jitter introduced. The significant performance degradation of NRZ, RZ and FSCR DACs has a good matching with the estimation given by Eq. (4). It also presents the same trend of jitter sensitivity as predicted by Eq. (1) for HRZ, SCR, and FSCR DACs. Fig 3 (c) shows the simulated SNDR under the influence of clock jitter for a fixed ELD of $10 \% T_{S}$. As previously analyzed, the error induced by the resetting is eliminated by introducing a certain ELD. It also indicates that the jitter induced IBN begins to dominate the noise contribution when $\sigma_{j}=0.01 \% T_{S}$ for RZ and HRZ; $\sigma_{j}=0.1 \% T_{S}$ for NRZ; and $\sigma_{j}=1 \% T_{S}$ for exponentially decaying SCRs.

To investigate the impact of DAC schemes on integrator nonidealities, the CT I $\Sigma \Delta$ ADC with different DACs is modeled in Verilog-A and simulated using Cadence Spectre. Only the $1^{\text {st }}$ integrator is examined here, since it generally consumes the highest power and errors entering this stage are not suppressed by noise shaping. The normalized unity-gainbandwidth (UGB), DC gain and transconductance needed for the feedback $\mathrm{Gm}$ cell $\left(G_{m, f b}\right)$ in the $1^{\text {st }}$ integrator, assuming a Gm-C implementation (as shown Fig. 1) are presented in Table I. The simulated SNDR under the influence of finite SR of the $G_{m, f b}$ in the $1^{\text {st }}$ integrator are compared in Fig. 4 (a) and (b) for rectangular DACs and exponentially decaying DACs, respectively. Simulation results confirm the previous theoretical analysis by showing that the rectangular DACs impose less design requirements on the $1^{\text {st }}$ integrator compared with the exponentially decaying DACs. Obviously, the price to pay for relaxed requirements, and consequently possible lower power consumption, is the increased sensitivity to timing error, especially clock jitter, according to Fig. 3 . 
TABLE I

UGB, GM AND DC GAIN IN THE $1^{\text {st }}$ INTEGRATOR FOR DIFFERENT DACS.

\begin{tabular}{cccc}
\hline \hline Feedback DACs & $U G B_{f b}$ & $G_{m, f b}$ & $A_{D C, f b}$ \\
\hline $\mathrm{NRZ}$ & $33.51 \% \times f_{S}$ & $1.07 \mu \mathrm{S}$ & $78.8 \mathrm{~dB}$ \\
\hline $\mathrm{RZ} / \mathrm{HRZ}$ & $67.03 \% \times f_{S}$ & $2.14 \mu \mathrm{S}$ & $84.8 \mathrm{~dB}$ \\
\hline $\mathrm{SCR}$ & $193.42 \% \times f_{S}$ & $6.19 \mu \mathrm{S}$ & $94.1 \mathrm{~dB}$ \\
\hline $\mathrm{FSCR}$ & $150.66 \% \times f_{S}$ & $4.82 \mu \mathrm{S}$ & $91.9 \mathrm{~dB}$ \\
\hline $\mathrm{SCSR}$ & $77.25 \% \times f_{S}$ & $2.47 \mu \mathrm{S}$ & $86.1 \mathrm{~dB}$ \\
\hline \hline
\end{tabular}

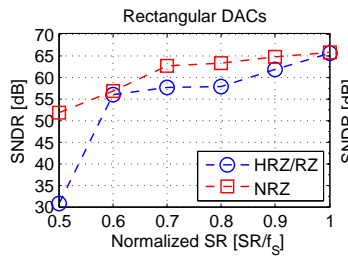

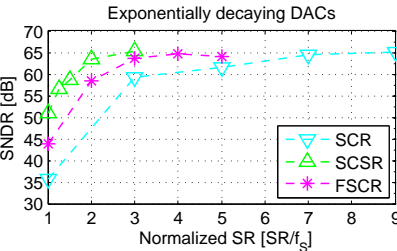

Fig. 4. Simulated SNDR with varying op-amp SR of the $G_{m, f b}$ in the first integrator. (a) rectangular DACs, and (b) exponentially decaying DACs.

As an example, the power spectral density (PSD) for each DAC scheme is presented in Fig. 5, where nonidealities (i.e., ELD, clock jitter and SR) are introduced for each case. While the ELD and clock jitter show their timing error sensitivity, the SR determines the biasing current needed in the opamp, which is an indicator of power consumption. RZ and HRZ are the most sensitive to clock jitter while their SR requirements are slightly higher than for NRZ. SCR imposes the most stringent SR requirement, which may not be very attractive option for this power-constrained application. SCSR achieves a good trade-off between timing error sensitivity and SR requirement, however, the complexity of its circuit implementation would introduce extra power consumption. In terms of timing error immunity, FSCR is the best, however, the speed requirement is the highest. In general, for the relatively low speed biosensor applications, NRZ appears a good candidate due to its best power efficiency. The target resolution is obtained with very low requirements on the $1^{\text {st }}$ integrator and acceptable clock jitter values. In addition, a NRZ feedback DAC can be easily implemented with standard circuitry. The resetting induced error can be avoided, for instance by guaranteeing a certain ELD. Using NRZ DAC results in inter-symbol interference (ISI) in traditional CT $\Sigma \Delta$ ADCs [11]. This is not a problem for the incremental mode, as each sample is converted independently. For the modulator topology shown in Fig. 1 in particular, there is another advantage of choosing the NRZ over other schemes. Only when using the NRZ DAC scheme, the quantization error of the CT I $\Sigma \Delta$ modulator is input signal independent [12].

\section{CONCLUSION}

In this paper, the impact of feedback DACs on the performance of CT incremental $\Sigma \Delta$ ADCs has been analyzed with an emphasis on biosensor applications. Different DAC schemes have been studied comparatively through theoretical
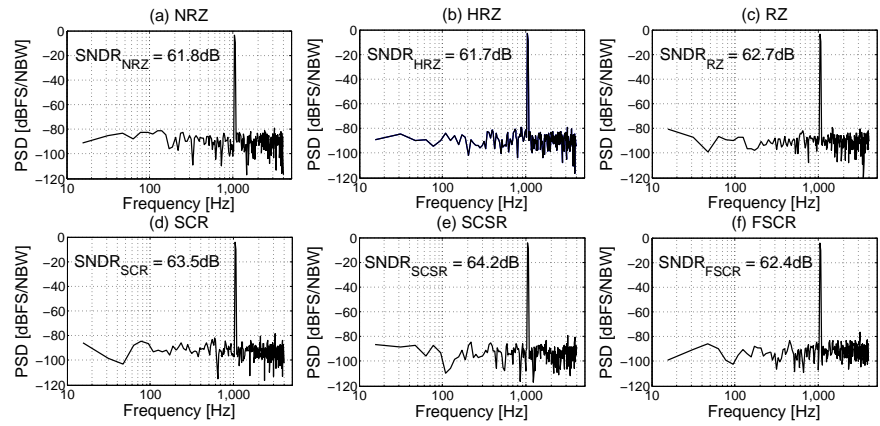

Fig. 5. PSD plots of the CT I $\Sigma \Delta$ ADC employing different DAC schemes $P_{\text {sig }}=-3.5 \mathrm{dBFS}, f_{\mathrm{sig}} \approx f_{B} / 4$, and $N_{\mathrm{FFT}}=512 . \tau_{d}=10 \% T_{S}$ for all DACs (a) $S R_{N R Z}=0.8 \times f_{S}, \sigma_{j, N R Z}=0.1 \% T_{S}$; (b) $S R_{H R Z}=1 \times f_{S}$, $\sigma_{j, H R Z}=0.02 \% T_{S}$; (c) $S R_{R Z}=1 \times f_{S}, \sigma_{j, R Z}=0.04 \% T_{S}$; (d) $S R_{S C R}=6 \times f_{S}, \sigma_{j, S C R}=0.1 \% T_{S}$; (e) $S R_{S C S R}=3 \times f_{S}$, $\sigma_{j, S C S R}=0.4 \% T_{S}$; and (f) $S R_{F S C R}=2 \times f_{S}, \sigma_{j, F S C R}=0.2 \% T_{S}$.

analysis and behavioral simulations. The criteria for comparison are the immunity to timing errors and the influence on circuit requirements. In addition, by considering a typical testcase, the feedback DAC schemes have been further discussed, aiming to provide hints on achieving a power aware solution.

\section{ACKNOWLEDGMENT}

The authors would like to thank the Swedish Research Council (VR) for funding this work.

\section{REFERENCES}

[1] J. Márkus, J. Silva, and G. C. Temes, "Theory and applications of incremental $\Sigma \Delta$ converters," IEEE Transactions on Circuits and Systems I: Regular Papers, vol. 51, no.4, 2004, pp. 678-690.

[2] J. Webster, Medical instrumentation: application and design. Wiley, 2009.

[3] J. Garcia, S. Rodriguez, and A. Rusu, "A Low-Power CT Incremental $3^{\text {rd }}$ Order $\Sigma \Delta$ ADC for bio-sensor Applications," IEEE Transactions on Circuits and Systems I: Regular Papers, vol. 60, no.1, 2013, pp. 25-36.

[4] M. Ortmanns, and F. Gerfers, Continuous-time sigma-delta A/D conversion: fundamentals, performance limits and robust implementations. Springer, 2006.

[5] J. Cherry and W. Snelgrove, Continuous-time delta-sigma modulators for high-speed $A / D$ conversion: theory, practice, and fundamental performance limits. Kluwer Academic, 2000.

[6] M. Ortmanns, F. Gerfers, and Y. Manoli, "A continuous-time $\Sigma \Delta$ modulator with reduced sensitivity to clock jitter through SCR feedback," IEEE Transactions on Circuits and Systems I: Regular Papers, vol. 52, no. 5, pp. 875-884, 2005.

[7] M. Anderson and L. Sundström, "Design and Measurement of a CT $\Sigma \Delta$ ADC With Switched-Capacitor Switched-Resistor Feedback," IEEE Journal Solid-State Circuits, vol. 44, no. 2, pp. 473-483, 2009.

[8] J. G. Jo, J. Noh, and C. Yoo, "A 20-MHz Bandwidth Continuous-Time Sigma-Delta Modulator With Jitter Immunity Improved Full Clock Period SCR (FSCR) DAC and High-Speed DWA,' IEEE Journal of Solid-State Circuits, vol. 46, no. 11, pp. 2469-2477, 2011.

[9] S. Tao, J. Garcia, S. Rodriguez and A. Rusu, "Analysis of Exponentially Decaying Pulse Shape DACs in Continuous-Time Sigma-Delta Modulators," in IEEE International Conference on Electronics, Circuits, and Systems, Dec. 2012, pp. 424-427.

[10] W. Yu, M. Aslan, and G. C. Temes, "82 dB SNDR 20-channel incremental ADC with optimal decimation filter and digital correction," in IEEE Custom Integrated Circuits Conference, Sept. 2010, pp. 1-4.

[11] L. Breems and J. Huijsing, Continuous-time sigma-delta modulation for $A / D$ conversion in radio receivers. Kluwer Academic Publishers, 2002.

[12] J. Garcia, S. Rodriguez, and A. Rusu, "On Continuous-Time Incremental Sigma-Delta ADCs with Extended Range," Transactions on Instrumentation and Measurement, vol. 62, no. 1, pp. 60-70, 2013. 